\title{
Deterministic and Probabilistic Dynamic Allowance for Railway Bridges from Measurement
}

\author{
L. Connolly ${ }^{1}$, E.J. OBrien ${ }^{2}$, A. O'Connor ${ }^{3}$, D. Hajializadeh ${ }^{4}$ \\ C. Leahy ${ }^{5}$ and C. Bowe
}

${ }^{1}$ Roughan \& O'Donovan Innovative Solutions, Dublin, Ireland

${ }^{2}$ University College Dublin, Ireland

${ }^{3}$ Trinity College Dublin, Ireland

${ }^{4}$ University of Surrey, Guildford, United Kingdom

${ }^{5}$ Arup, Dublin, Ireland

\begin{abstract}
In a traditional deterministic assessment, a dynamic amplification factor (DAF) is applied to the static loading in order to account for dynamics. The codified DAF values are appropriately conservative in order to consider the wide range of structures and load effects to which they are applied. In the current analysis, a site-specific assessment dynamic ratio (ADR) is calculated from direct measurement on an eighty year old steel truss railway bridge for two specific elements. The ADR is defined as the ratio of characteristic total stress to the characteristic static stress. The application of ADR is a relatively new concept which has rarely been considered for railway bridges. An assessment performed on the bridge in question showed a decrease in the dynamic allowance when considering the site specific ADR, resulting in a decrease in calculated stress of up to $26 \%$. The measurements available were also used to derive a robust stochastic model for dynamic allowance which considered the correlation between DAF and stress level. The developed model was applied to a probabilistic assessment and resulted in an increase in calculated reliability of $12 \%$ for the rail bearers and $5 \%$ for the cross girders.
\end{abstract}

Keywords: dynamics, DAF, ADR, truss, railway, bridge, probabilistic, assessment, reliability, monitoring, vibration, signal, filter.

\section{Introduction}

In order to account for dynamics in bridge design and assessment, a Dynamic Amplification Factor (DAF) is typically applied to static loading [1]. This is defined as the ratio of total (static + dynamic) load effect, $L E_{\text {Total }}$, to the static load effect, $L E_{\text {Static }}$, for a particular event [2]:

$$
D A F=\frac{L E_{\text {Total }}}{L E_{\text {Static }}}
$$


It has been shown that in many cases the factors employed have been overconservative [3]. This is considered to be acceptable for bridge design codes in order to consider the wide range of structures and load effects to which they are applied. However, for existing structures, this conservatism can result in costly and unnecessary repairs being put in place. Previous research has shown that the DAFs defined in most codes are appropriate for lower levels of loading, such as single event crossings with light trucks on road bridges [3]. However, for heavier loads, measured DAFs have been shown to be significantly lower [3]. One possible reason for this is the speed reduction associated with heavy vehicles, which often reduces dynamic amplification as noted by Frýba [4]. The formulation of DAF in Equation 1 also fails to account for the probability of maximum static and total load effects occurring for separate events. The Danish Roads Directorate (DRD) document on reliability based classification of the load carrying capacity of existing road bridges [5], suggests the use of a stochastic increment for dynamic amplification for gross vehicle weight (GVW) modelled as a normally distributed variable with mean and standard deviation equal to $41.5 / \mathrm{GVW}$, where the GVW is in $\mathrm{kN}$. The result of this model is that dynamics reduce with increasing vehicle weight, as shown in Figure 1.

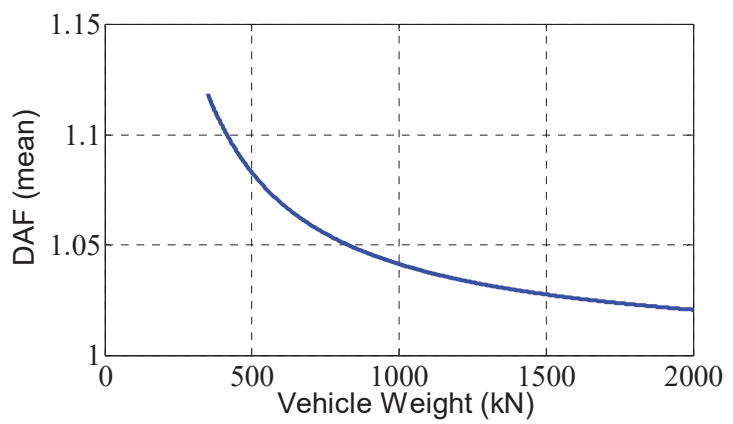

Figure 1: Mean DAF versus vehicle weight from Danish Roads Directorate [5]

In order to alleviate the drawbacks associated with traditional codified DAFs, various authors [6,7] have introduced the concept of an Assessment Dynamic Ratio (ADR), defined as the ratio of the characteristic total (static + dynamic) load effect, $\widetilde{L E}_{\text {Total }}$, to the characteristic static load effect, $\widetilde{L E}_{\text {Static }}$.

$$
A D R=\frac{\widetilde{L E}_{\text {Total }}}{\widetilde{L E}_{\text {Static }}}
$$

This paper is based upon Connolly et al. [9] but presents results for a significantly larger database of measurement consisting of 35 days of recording and 724 train loading events. In addition, the results are considered for both rail bearers and cross girders, where the previous research considered rail bearers only. The sensitivity of ADR to the chosen return period has also been addressed and the derivation of the stress-varying probabilistic distribution for dynamic allowance has been described. 


\subsection{Calculation of ADR}

In order to evaluate the ADR for a specific bridge, the characteristic total and characteristic static load effects are required. OBrien et al. [6] used the output from a Bridge Weigh-in-Motion (WIM) system to obtain the strain signals associated with total (static + dynamic) strain. Characteristic total strains were calculated by statistical extrapolation. The WIM system used also calculates a 'measured' static influence line for the structure. This was used in conjunction with the measured axle weights to infer the strains associated with the static response. The characteristic total and static strains were then used to calculate the ADR. A disadvantage in the procedure used by OBrien et al. [6] is that the inaccuracies associated with calculation of axle weights using bridge WIM result in the introduction of a possible bias in the calculation of ADR. A similar procedure is used in SAMARIS [3]. In each case, an additional disadvantage is the requirement of cumbersome calibration of the Bridge WIM system, which is not required for the approach adopted in this paper.

Simulation can also be used to calculate characteristic load effects for both static and dynamic response. The research performed as part of SAMARIS [3] demonstrated this. Once again, the conclusion was drawn that the dynamic amplification reduces as the magnitude of the load effect increases. Despite the advantages associated with site-specific ADR calculation, the theory has rarely been applied to railway bridges. One possible reason for this is the lack of variation in the distribution of trains that pass over a specific bridge, making it difficult to perform meaningful statistical extrapolations [7]. The procedure was first introduced for railway bridges by Cantero et al. [8], where it was concluded that a smaller recommended allowance for dynamics can be safely accommodated for various bridge configurations. Cantero et al. [8] calculated characteristic load effects by performing Monte Carlo simulation on a traintrack-bridge interaction model, accounting for track irregularities. The bridge model considered was a 2-dimensional Euler-Bernoulli beam. Considering the number of inputs and the large amount of computing power required for this type of simulation, it may be difficult to perform the analysis on a more complex 3-dimensional bridge model. Therefore, a different approach is proposed in this paper.

Structural health monitoring (SHM) has become an increasingly important technology to determine the static and dynamic responses of infrastructure during construction or while in service $[10,11,12]$. In this paper, a case study bridge (see Section 3.1) is used to calculate a site-specific ADR, incorporating a SHM system. Strain signals from the bridge, measuring the total (static + dynamic) strain, are filtered using band-pass filtering algorithms (see Section 2) to remove dynamics. This procedure was also demonstrated by Lynch and Loh [13] who used a low-pass Butterworth signal filter to remove high-frequency noise. In this paper, maximum daily strains will be used to extrapolate characteristic total and characteristic static strains. The site specific ADR will then be calculated for two critical members. The implications of the calculation will be demonstrated in terms of both a deterministic and probabilistic assessment. 


\section{Demonstration of concept: filtering of dynamics}

In order to demonstrate and validate the methodology employed to filter the dynamic response, consider a simply supported beam of length, $L$, subjected to a moving point load, $P$, moving at a speed, $c$, as shown in Figure 2. Neglecting vehicle mass, track profile, etc., the dynamic displacement response of the beam was formulated by Frýba [4]:

$$
\begin{aligned}
v(x, t)=v_{o} & \sum_{j=1}^{\infty} \frac{1}{j^{2}\left[j^{2}\left(j^{2}-\alpha^{2}\right)^{2}+4 \alpha^{2} \beta^{2}\right]}\left[j^{2}\left(j^{2}-\alpha^{2}\right) \sin j \omega t\right. \\
& -\frac{j \alpha\left[j^{2}\left(j^{2}-\alpha^{2}\right)-2 \beta^{2}\right]}{\left(j^{4}-\beta^{4}\right)^{1 / 2}} e^{\omega_{b} t} \sin \omega_{(J)}^{\prime} t \\
& \left.-2 j \alpha \beta\left(\cos j \omega t-e^{-\omega_{b} t} \cos \omega_{(J)}^{\prime} t\right)\right] \sin \frac{j \pi x}{L}
\end{aligned}
$$

where

$$
\begin{aligned}
& \alpha=\frac{c l}{\pi}\left(\frac{\mu}{E J}\right)^{1 / 2} \\
& \beta=\frac{\omega_{b} l^{2}}{\pi^{2}}\left(\frac{\mu}{E J}\right)^{1 / 2}
\end{aligned}
$$

and

$x=$ length coordinate with origin at the left hand end of the beam,

$t=$ time since the force arrived on the beam,

$v(x, t)=$ vertical displacement at point $x$ and time $t$,

$j=$ mode of vibration,

$\omega=$ circular frequency of damping of the beam,

$E=$ Young's modulus of the beam,

$J=$ constant $2^{\text {nd }}$ moment of area of the beam cross section,

$\mu=$ constant mass per unit length of the beam.

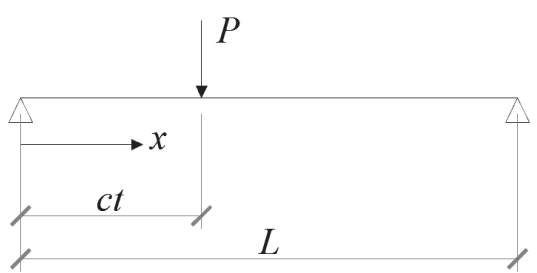

Figure 2: Simply supported beam subjected to a moving point load

The result is calculated for a $20 \mathrm{~m}$ span concrete beam with a breadth of $1 \mathrm{~m}$ and a depth of $0.8 \mathrm{~m}$. A $200 \mathrm{kN}$ point load is considered with a velocity of $20 \mathrm{~m} / \mathrm{s}$. By computing the first derivative of Equation 3 with respect to $x$, the dynamic strain response can be derived for the beam due to the moving load. Figure 3 illustrates the 
total (dynamic + static) strain at the centre of the beam in comparison to the static response. For this simple example, the dynamic increment may be considered as the ratio of the maximum total (dynamic + static) and maximum static strain, which corresponds to a value of 1.14. A total of fifty modes of vibration are considered in Figure 3, although the result is approximately the same for around ten modes, indicating that the majority of vibration is associated with the first few modes. By computing the second derivative of Equation 3 with respect to time, the acceleration response of the beam can be obtained. A Fourier transform can then be applied to the acceleration signal to obtain the natural frequencies of the beam. These can be compared to the theoretical natural frequencies of a simply supported beam (Figure 4). The $j^{\text {th }}$ natural frequency of this beam, as formulated by Frýba [4], is calculated as:

$$
\alpha=\frac{j^{2} \pi}{2 L^{2}}\left(\frac{E J}{\mu}\right)^{1 / 2}
$$

Figure 4 illustrates the first five natural frequencies of the beam, computed by Fast Fourier Transform (FFT), compared to the theoretical frequencies. The second and fourth frequency cannot be seen in Figure 4 for the FFT. This is because the theoretical sensor location is at the centre of the beam, where there is no excitation by the second or fourth frequencies.

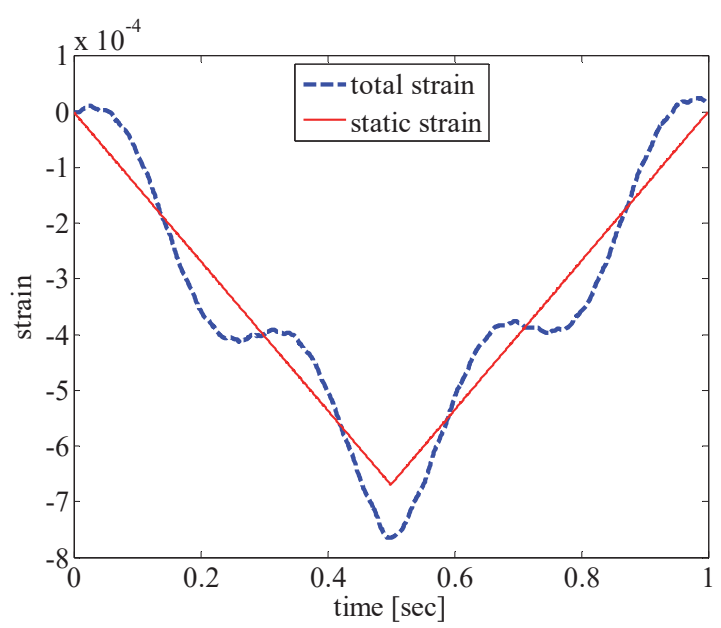

Figure 3: Total (static + dynamic) and static strain response at the centre of the beam formulated as per Equation 3

In practice, the dynamic strain is measured by the sensor on the bridge. As stated above, in order to calculate the ADR, both the static and dynamic responses are required. The static response is obtained by applying a filter to the total (dynamic + static) response. Digital filters are often employed to remove specific frequency domains from an input signal [15]. This is achieved by passing the input signal through a low-pass filter. This attenuates the parts of the input signal which relate to 
frequencies above a certain value, referred to as the "cut-off" of the filter [14]. In this section, the portion of the total (dynamic + static) strain signal which relates to dynamic vibration is effectively removed by applying a low pass filter. The remaining part of the signal, that static portion, is substantially unaffected. A low-pass Butterworth signal filter was chosen as it has low ripple in the pass band and stop band, meaning that the static response is not altered [15]. Of course, a critical input to the filter is the first natural frequency, as this defines the cut-off. Vibration associated with higher frequencies will also be filtered out of the total response, although their effect on the dynamic response is expected to be low.

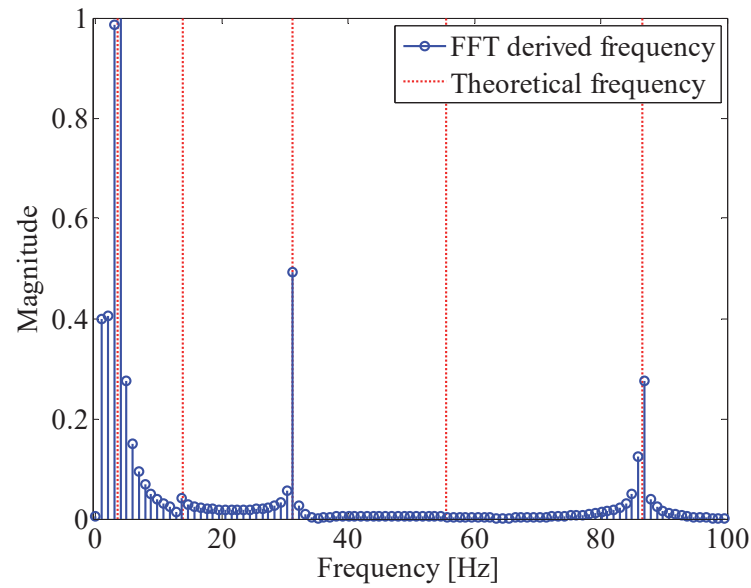

Figure 4: Theoretical and FFT computed natural frequencies at the centre of the beam formulated as per Equation 3

As Butterworth signal filters have a slower roll-off than, for example, Chebyshev signal filters $[15,16]$, the cut-off was set at a value $10 \%$ below the first natural frequency of the beam, to ensure that all vibration associated with the first natural frequency was removed. Figure 5 shows the result of passing the total strain signal from Figure 3 through a $7^{\text {th }}$ order low-pass Butterworth filter. It is clear that the majority of the dynamic oscillation has been removed from the total strain signal, and the ratio of the unfiltered to the filtered signal is an accurate approximation of DAF.

\section{Calculation of site-specific Dynamic Allowance for the Boyne Viaduct}

\subsection{Assessment}

The Boyne Viaduct (Figure 6) in Drogheda, Co. Louth, Ireland, is around 86 years old and consists of three steel spans of 40, 80 and 40 metres. Only the central span was considered for this study. The riveted steel truss structure has an arched profile consisting of ten bays with cross beams spanning between the node points of the truss. 
The ballasted track is supported on a steel deck plate over the rail bearers, which span between the cross beams.

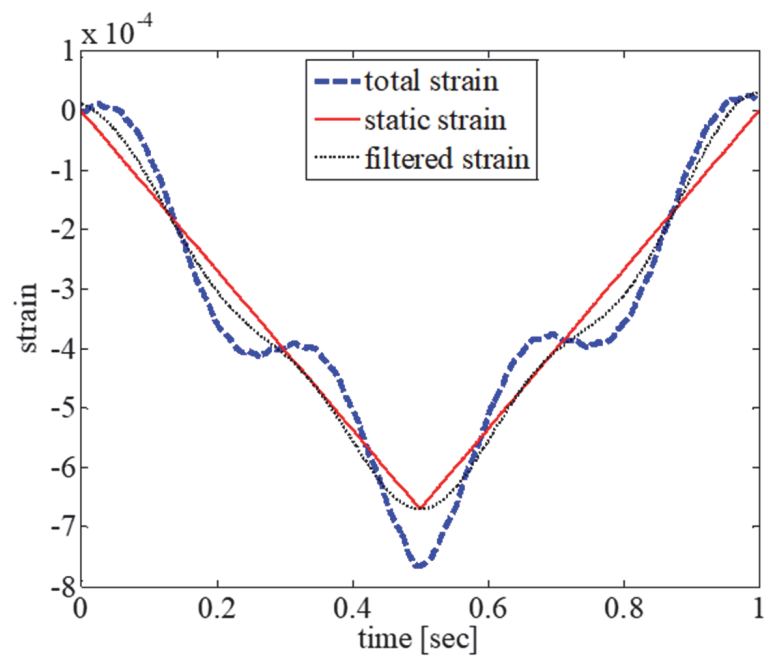

Figure 5. Filtering of total (static + dynamic) strain response for theoretical beam

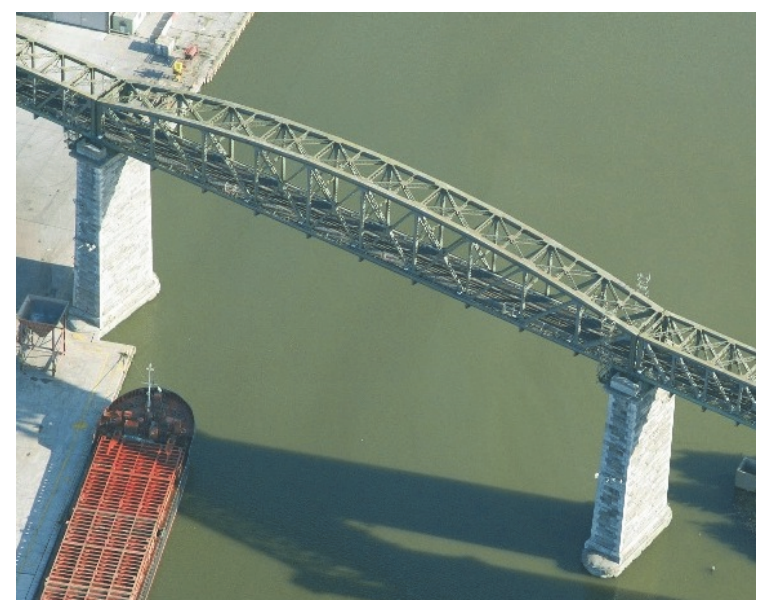

Figure 6: Central span of the Boyne Viaduct, Drogheda, Co. Louth, Ireland.

A finite element (FE) model was developed and is illustrated in Figure 7. The model was used to perform an ultimate limits state (ULS) deterministic assessment of the structure in accordance with the UK design manual for roads and bridges (DMRB, $[17,18])$. A full description of the assessment can be found in Connolly et al. [19]. 
The rail bearers of the structure were the only elements to fail the assessment. The load effect in question was ULS yielding and the over-stress was 6\%. The utilisation of the cross beams corresponded to a $21 \%$ under-stress.

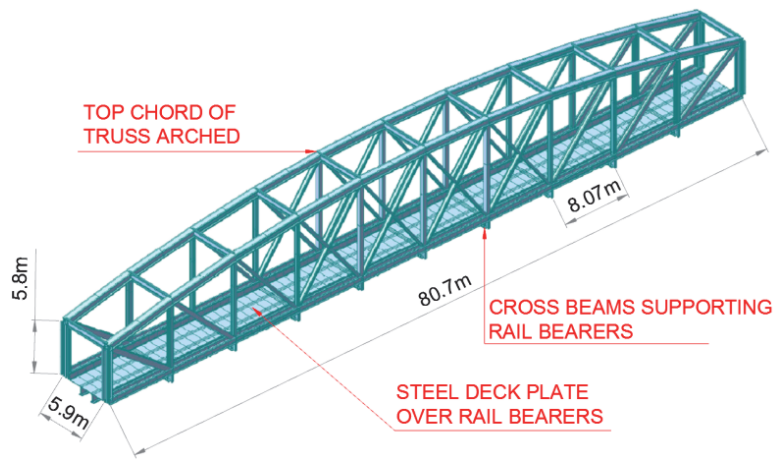

Figure 7: Finite element model of the Boyne Viaduct central span [20]

The member sections for the top and bottom chord of the bridge, as well as the rail bearers and cross girders are illustrated in Table 1. All members are formed as builtup rivetted sections, with the truss elements also incorporating steel lacing.

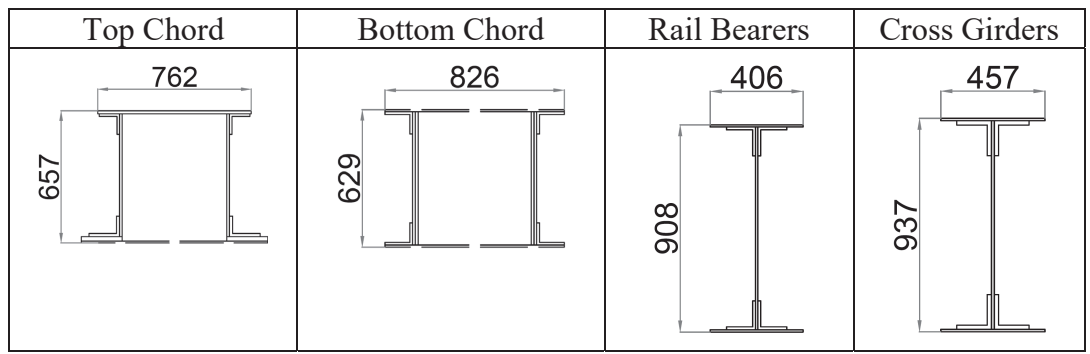

Table 1: Member sections for elements on the Boyne Viaduct

After having failed the deterministic assessment, the rail bearers of the structure were assessed probabilistically under ULS yielding. The cross girders were also assessed probabilistically for completeness. The majority of the stochastic modelling was performed in accordance with the guidelines of the Danish Roads Directorate [5]. Full details of the probabilistic assessment can be found in [19]. FORM analysis $[21,22]$ was used to compute the reliability index, $\beta$, for the rail bearer. The analysis converged to a value of $\beta=4.56$. The target level of reliability may be specified by the client, but ISO 2394:1998 [23] recommends a value of 3.8 for a "moderate" cost of safety measures and a "great" consequence of failure. The corresponding level recommended by the Joint Committee on Structural Safety is 4.4 [24]. A reliability index of 5.66 was calculated for the cross girders 
Within the deterministic assessment, the dynamic allowance was calculated from the guidelines in the DMRB [17]. Values of 1.42 and 1.25 were calculated for the rail bearers and cross girders, respectively. As stated in Section 1, this DAF may be inappropriately conservative for the structure in question. For the probabilistic assessment, the DAF was modelled in a manner similar to the DRD guidelines [5] as follows:

$$
D A F=1+\varepsilon
$$

The dynamic increment $(\varepsilon)$ was modelled as a normally distributed variable with a coefficient of variation (CoV) of 1.0 as recommended by the DRD [5]. However, the mean value specified by the DRD [5] relates to road bridges. Therefore, the mean value was determined as that which resulted in a $98 \%$ fractile value equal to the deterministic increment ( 0.42 for the rail bearers and 0.25 for the cross girders), in line with work by O'Connor et al. [25]. This resulted in a normally distributed variable for the $\varepsilon$ increment with mean and standard deviation equal to 0.14 for the rail bearers and 0.08 for the cross girders. It is clear that the stochastic modelling of dynamic allowance considered here was based on codified loading, and does not take account for the negative correlation between vehicle weight and DAF, as noted in [3]. The remaining sections of this paper describe how SHM was used to calculate the DAF as defined in Equation 7.

The assessment performed was used to define an SHM strategy for the structure. Four rosette strain gauges were placed on the bottom flanges of the critical rail bearers and the most critical cross beam. In addition to the work performed in this paper, these gauges will be used for model validation and fatigue analysis. The bottom chord of the truss was instrumented with triaxial accelerometers. Figure 8 shows accelerometers and strain gauges installed on the structure.

(a)

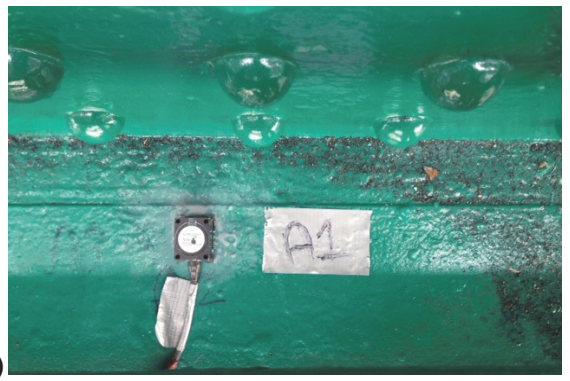

(b)

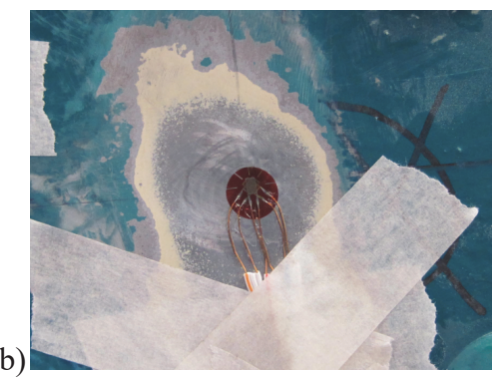

Figure 8. Sensors on the Boyne Viaduct: (a) accelerometer and (b) strain gauge

\subsection{Evaluation of natural frequencies}

In order to identify the optimum locations for the accelerometers on the Boyne viaduct, a modal analysis was carried out on the FE model. Elevations of the first two vertical mode shapes of the structure are illustrated In Figure 9. Their respective frequencies of vibration were calculated as 3.9 and 9.2 Hz. Based on the mode shapes, 
accelerometers were placed at centre span and at one third span. Vibration responses from the accelerometers are illustrated in Figure 10.

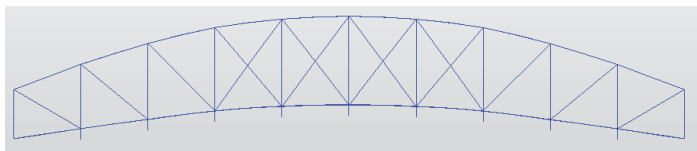

(a)

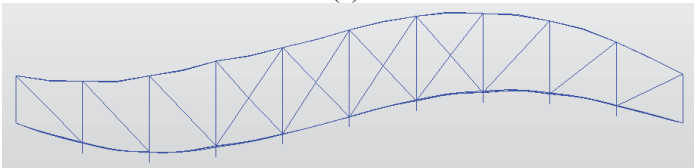

(b)

Figure 9: Vertical mode shapes of the Boyne Viaduct: (a) first and (b) second

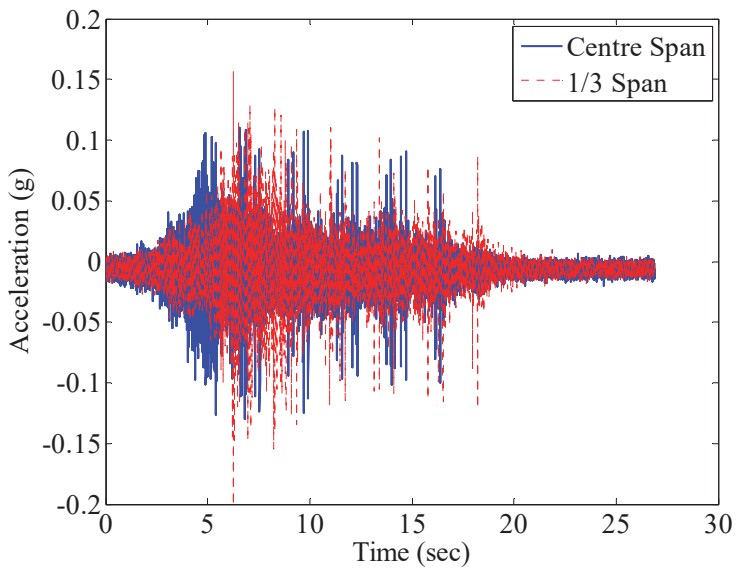

Figure 10: Vertical acceleration at centre span and one third span of the Boyne Viaduct

As stated in Section 2, a critical input to the low-pass filter is the natural frequency of vibration of the structure. FFTs were applied for the acceleration responses and are illustrated in Figure 11. It should be noted that the Fourier transforms shown in Figure 11 are based on all signals (from all 724 train passage events) together. It is clear that for both FFTs, there is a peak around $10 \mathrm{~Hz}$. Most of the FFTs for each individual event also showed a peak at this frequency. Therefore, this was the cut-off defined for the low-pass filter. Each FFT also showed a sharp peak at a frequency of approximately zero $\mathrm{Hz}$, which is related to the static response. Although there are peaks present close to the calculated first and second natural frequencies of $3.9 \mathrm{~Hz}$ and $9.2 \mathrm{~Hz}$, the peaks are not very clear due to other frequencies being in place in the data (e.g. due to sensor noise, trains, interference etc.). A better picture may be obtained by performing some pre-processing of the data to remove parts of the vibration which 
correspond to sensor noise/trains etc. However, this was not required for the current study.

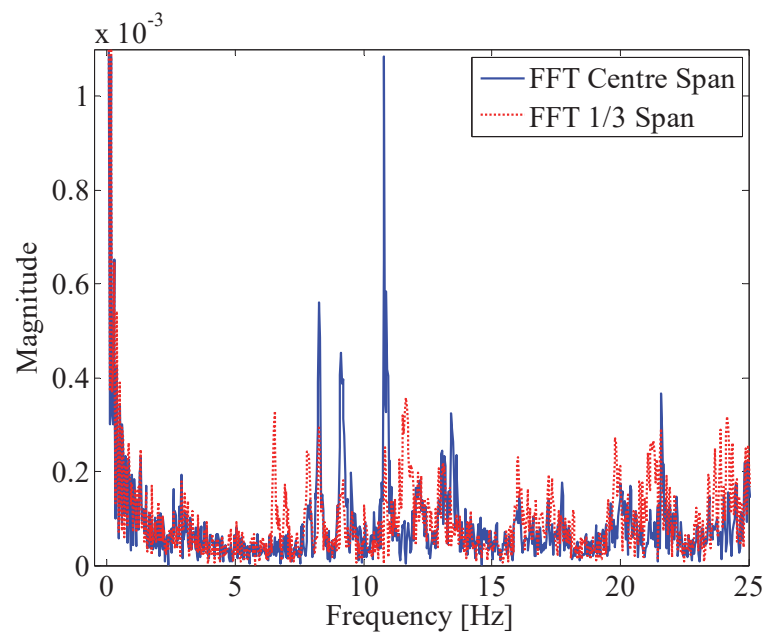

Figure 11: FFT of vertical acceleration at centre span and one third span of the Boyne Viaduct

\subsection{Removal of dynamics}

In order to remove the dynamics from the total (static + dynamic) portion of the strain signals for the centre of the rail bearer and cross girder, the low-pass filter discussed in Section 2 was applied to the strain signals. Various levels of dynamic amplification were noted for different events. Figure 12(a) shows an example with a maximum DAF (per train) of 1.07, while Figure 12(b) shows an example with a maximum DAF (per train) of 1.02. The figures also show a magnified view of selected peaks. It should be noted that the strain signals shown are principal strains for the element in question, which are considered to be appropriate for the critical yield criterion in the assessment. These are calculated from each strain gauge reading, $\varepsilon_{i}$, of a stacked rectangular rosette using Equation 8 [26].

$$
\frac{\varepsilon_{1}+\varepsilon_{3}}{2} \pm \frac{1}{\sqrt{2}} \sqrt{\left(\varepsilon_{1}-\varepsilon_{2}\right)^{2}+\left(\varepsilon_{2}-\varepsilon_{3}\right)^{2}}
$$

It is clear from Figure 12 that the DAF considered in the DMRB is conservative for the bridge in question as the maximum DAF detected in the monitoring period is $24 \%$ below the DAF recommended in the DMRB [17]. This is expected, considering that the age of the structure may indicate that it is over-designed. In addition, the ballasted track may tend to reduce dynamic amplification for the rail bearers, as noted in the Eurocode [27]. The authors also note that there is a speed restriction on the bridge of $50 \mathrm{~km} / \mathrm{hr}$, which will also tend to result in low levels of dynamic amplification as suggested by Frýba [4]. 


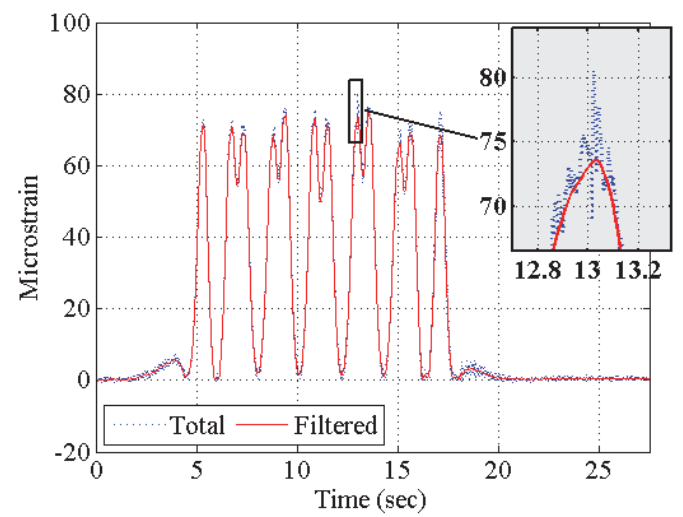

(a)

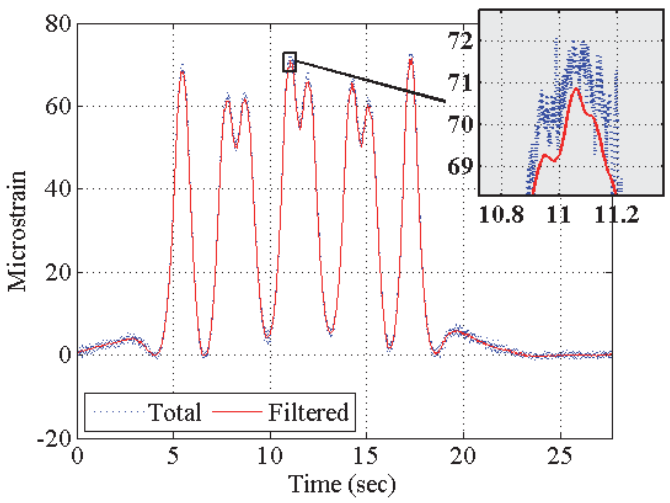

(b)

Figure 12: Low-pass filter applied to strain data at centre of rail bearer: (a) DAF = 1.07; (b) $\mathrm{DAF}=1.02$

\subsection{Calculation of dynamic allowance}

\subsubsection{Assessment Dynamic Ratio (ADR)}

In order to evaluate the characteristic ADR from Equation 2, the characteristic total (static + dynamic) load effect, $\widetilde{L E}_{\text {Total }}$, and the characteristic static load effect, $\widetilde{L E}_{\text {Static }}$, are required. In order to derive characteristic load effects, a Generalised Extreme Value (GEV) distribution is fitted to the maximum daily strains by maximum likelihood estimation. 35 days of measurement were available at the time of submission, consisting of 724 train passing events. The characteristic load effect is calculated at the appropriate return period. A return period of 1000 years is taken in the Eurocode for design [27]. A 75-year return period is considered here for an 
existing bridge. Maximum-per-day static and total strains are plotted on Gumbel probability paper in Figure 13. Gumbel probability paper is a double log plot of the cumulative distribution of the data. The result is that Gumbel distributed data appears as a straight line [21]. The 75 year return period for maximum daily values (365 days in a year) is given as:

$$
-\ln (-\ln (1-1 /[75 * 365])) \approx 10.22
$$

While previous research [6] suggests using 250 days per year when considering road traffic, this is not appropriate for railway bridges which carry passenger traffic only. In Figure 13 the dashed curve represents the GEV fit to the tail of the static strain, while the solid curve represents the GEV fit to the tail of the total (static + dynamic) strain. In order to assess the effect of the chosen return period, ADR values were calculated for return periods of 10 to 100 years. This showed that the choice of return period was insignificant with a variation in ADR of only $0.03 \%$.

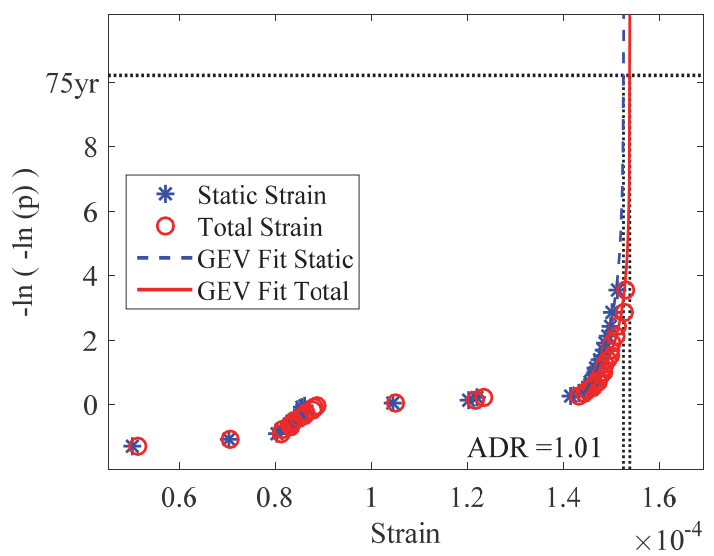

Figure 13: Calculation of ADR for rail bearers

The concave upward shape of the graph in Figure 13 indicates Weibull behaviour [21]. The ADR is calculated as 1.01. However, in order to allow a margin of safety, an ADR of 1.05 is suggested. This is considered for deterministic assessment in Section 3.4.4. The analysis was also performed for the cross girders of the structure. A similar ADR was calculated for these members as illustrated in Figure 14.

\subsubsection{DAF distribution}

For the probabilistic assessment, a distribution of dynamic amplification is required. In order to investigate an appropriate distribution, a histogram of all recorded dynamic amplifications (for individual events) is plotted in Figure 15. Normal and Lognormal distributions are fitted to the data. DAF is usually modelled as a normally distributed variable [5]. However, it is clear from the figure that the lognormal distribution provides a better fit to the data. This was confirmed with a goodness of 
fit test. This is to be expected for railway bridges, due to the length of the trains. While the effect of dynamics may reduce the total stress for road bridges, this is unlikely for train passages as the dynamic effect of one of the carriages is likely to have a constructive effect on stress. It should be noted that the distributions were fitted to the dynamic increment, $\varepsilon$, and not the DAF. The lognormal distribution ensures only positive values are considered for $\varepsilon$. It may also be concluded here that the assumption of the DRD guidelines [5] that the coefficient of variation in DAF is equal to 1.0 may not be appropriate for railway structures. This is also expected, considering the low standard deviation in railway traffic weights [28]. These results were also noted for the cross girders of the structure.

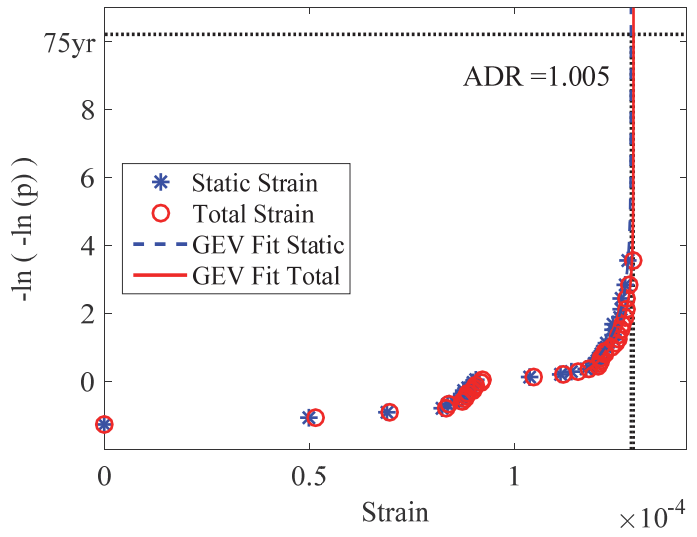

Figure 14: Calculation of ADR for cross girders

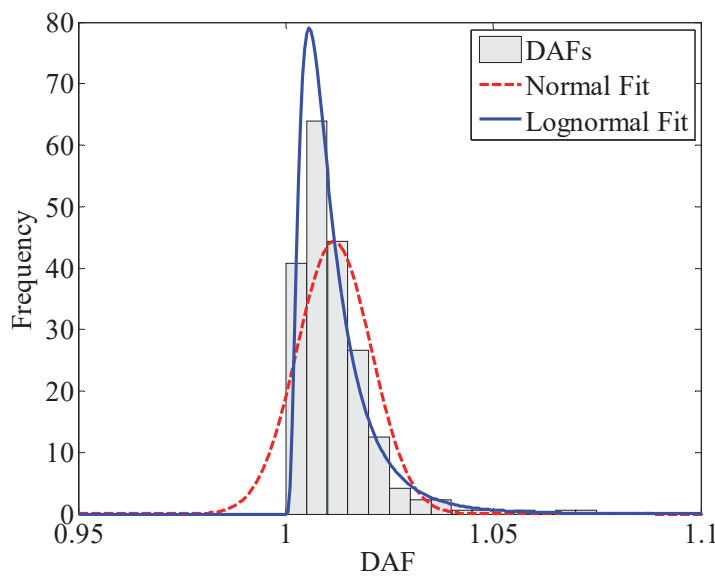

Figure 15: Fit of probability distributions to dynamic amplification 


\subsubsection{Stress varying DAF distribution}

The distribution fitted to the data in Section 3.4.2 is conservative, as consideration is not given to the trend of high dynamic amplification corresponding to lower stresses [3]. For this reason, a stress varying lognormal distribution is fit to the DAFs of Figure 15. The principal stress is calculated from Equation 10 [26]. In this equation, $E$ and $v$ are the Young's modulus and Poisson's ratio of steel, respectively. The $\varepsilon_{i}$ values correspond to the strains on each of the three gauges of the stacked rectangular rosette used.

$$
\frac{E}{2}\left(\frac{\varepsilon_{1}+\varepsilon_{3}}{1-v} \pm \frac{1}{1+v} \sqrt{\left(\varepsilon_{1}-\varepsilon_{2}\right)^{2}+\left(\varepsilon_{2}-\varepsilon_{3}\right)^{2}}\right)
$$

For the stress varying fit, an approach similar to that used by OBrien et al. [29] is adopted, who used a time-varying distribution of traffic loading to model growth in traffic loading over time. It is important to understand that this is not a bivariate statistical distribution but rather a one-dimensional distribution that changes with stress. The distribution is described by four parameters: $m_{\mu}, C_{\mu}, m_{\sigma}$ and $C_{\sigma}$. Maximum likelihood estimation [30] is then used to calculate the parameters $\mu_{i}$ and $\sigma_{i}$ of the lognormal distribution at each increment in stress $\left(\gamma_{i}\right)$ :

$$
\begin{aligned}
& \mu_{i}=m_{\mu} \gamma_{i}+C_{\mu} \\
& \sigma_{i}=m_{\sigma} \gamma_{i}+C_{\sigma}
\end{aligned}
$$

The stress varying fit to dynamic amplification is illustrated in Figure 16 for the rail bearers and cross girders. It is clear in Figure 16 that the inverse relationship between stress and DAF that was found in the literature [3] was also noted in the current research.

A $3 \mathrm{D}$ view of the stress varying distribution is shown for the cross girders in Figure 17 for illustration. It is clear that the standard deviation reduces as the stresses increase.

\subsubsection{Implications for safety assessment}

The ADR calculated in Section 3.4.1 is considered applicable to the deterministic assessment. The value of 1.05 adopted in this study for both the rail bearers and cross girders is considerably greater than that which was calculated. It is, however, still $26 \%$ below the value determined from the procedure outlined in the DMRB [17] for the rail bearers and $16 \%$ below the value calculated for the cross girders. The result is a reduction in the $6 \%$ over-stress previously noted for the rail bearers being reduced to a $20 \%$ under-stress. It is clear that this would obviate the need for further assessment. The effect for the cross girders is a reduction from a $21 \%$ under-stress to a $32 \%$ understress. 


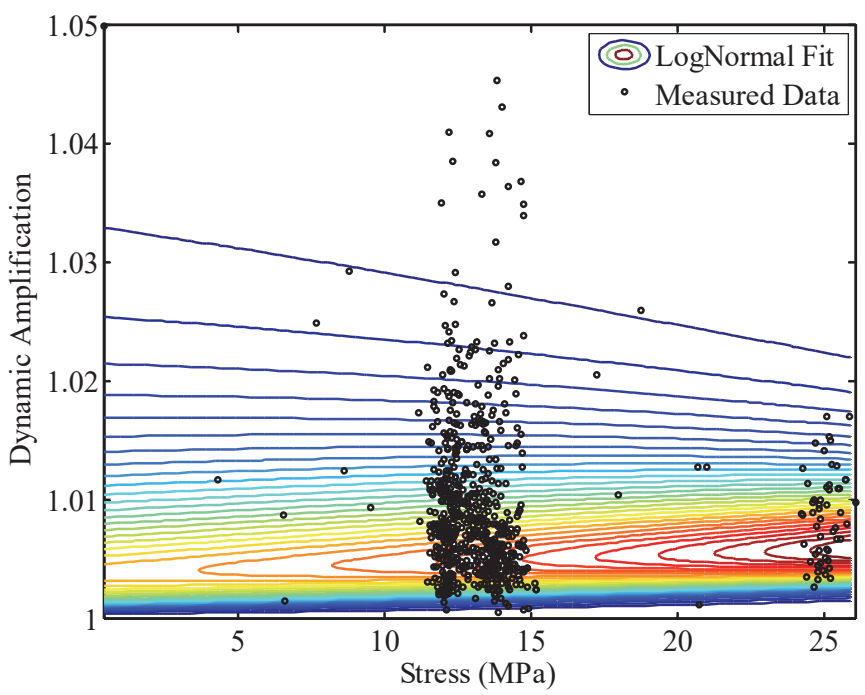

(a)

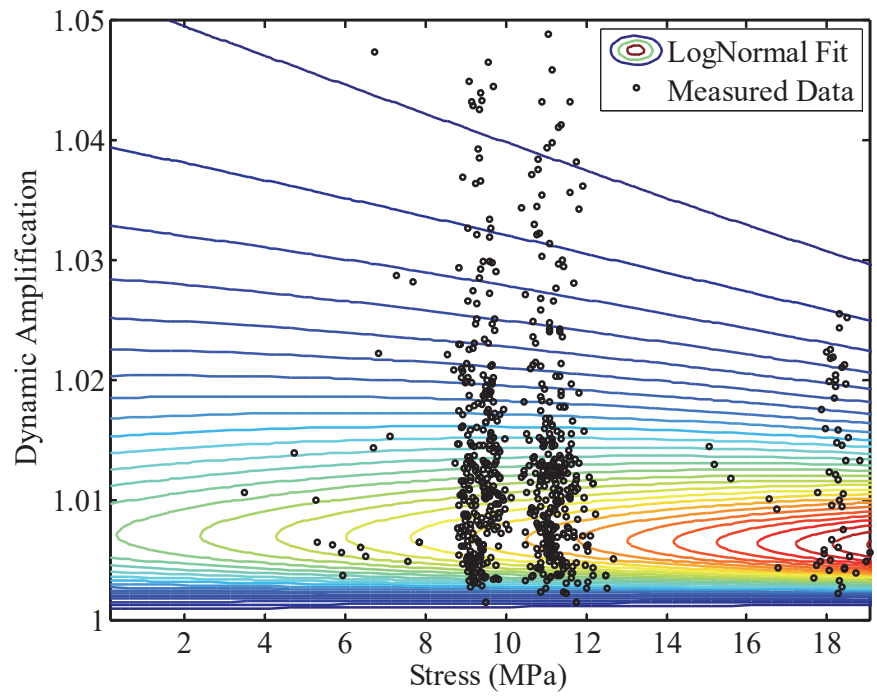

(b)

Figure 16: Stress varying lognormal distributions for dynamic amplification of (a) rail bearer and (b) cross girder 


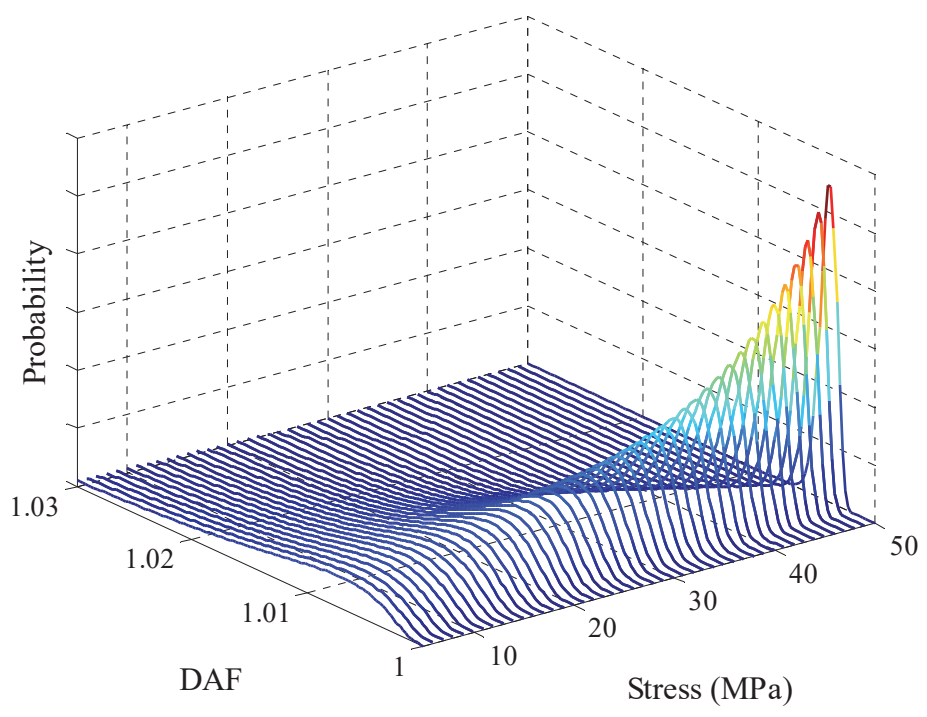

Figure 17: Three-dimensional plot of stress varying lognormal distribution for dynamic stress amplification of cross girder

In the probabilistic assessment, all parameters which relate to the load and resistance equation at ULS were modelled stochastically. This means that the static analysis was run 100,000 times, varying each parameter by randomly sampling from a distribution with a mean value $(\mu)$, and standard deviation $(\sigma)$. A Matlab random number generator was used to perform the sampling. The stress varying distribution derived in Section 3.4.3 allowed sampling from a separate distribution for DAF, depending on the stress level. This procedure resulted in an increase in the $\beta$-value from 4.56 to 5.10 for the rail bearers and from 5.66 to 5.95 for the cross girders.

\section{Conclusions and recommendations}

A deterministic and subsequent probabilistic assessment were carried out on an 80 year old riveted steel truss railway bridge. The bridge was shown to have an insufficient capacity under ULS yielding. Data from instrumentation on the bridge was used to derive a more appropriate dynamic allowance. The assessments were then re-formulated with consideration of the new representation of dynamic allowance.

The traditional DAF defined in the DMRB was replaced with the Assessment Dynamic Ratio (ADR). The application of ADR resulted in a reduction in the utilisation for the critical element and limit state from $6 \%$ over-stress to $20 \%$ under- 
stress. Therefore, the procedure developed shows the bridge to have sufficient capacity prior to the undertaking of a probabilistic assessment.

A stress varying distribution was derived for DAF and this was considered in the probabilistic assessment. This was shown to result in an increase in reliability of $12 \%$ from the original calculation for the rail bearers and 5\% for the cross girders.

The procedure developed herein is considered to have significant advantages over previous methods developed in the literature. For example, the expensive installation of a WIM system (and associated error) is avoided. In addition, the development of complex and intensive dynamic interaction FE models is not required. Indeed, the procedures developed herein may be applied ad-hoc to any structure with a sufficient structural health monitoring system installed.

The results showed that the calculated ADR for the assessed bridge is very small. This may be due to the very low speed restriction for trains crossing the bridge $(50 \mathrm{~km} / \mathrm{hr})$. This may not be the case however for all railway bridges. In fact, with the significant increase in high speed rail across Europe, dynamic amplification levels may be found to increase, inducing over-stresses in the future. The methodology developed here can be applied to different, more severe dynamic loading scenarios for railway bridges. Therefore, the authors recommend that it would also be beneficial to apply the method herein to other structures with higher levels of dynamic amplification in order to consider a wider range for the stress varying DAF distribution.

\section{Acknowledgements}

The research leading to these results was part of the DESTination RAIL project, a project funded by the EU Horizon 2020 Programme under call H2020-MG-2014 Mobility for Growth. Grant agreement no: 636285. The authors also gratefully acknowledge Iarnród Éireann, for facilitating the research associated with the Boyne Viaduct.

\section{References}

[1] D. Cantero, A. González, E.J. OBrien, "Comparison of bridge dynamic amplification due to articulated 5-axle trucks and large cranes", The Baltic Journal of Road and Bridge Engineering. 6(1), 39-47, 2011.

[2] D. Cantero, R. Karoumi, E.J OBrien, "Maximum total load effects in vehiclebridge dynamic interaction problems for simply supported structures", Proceedings of the 9th International Conference on Structural Dynamics, A. Cunha, E. Caetano, P. Ribeiro, G. Müller (Editors), EURODYN 2014. Porto, Portugal, 30 June - 2 July 2014

[3] SAMARIS report D30, "Guidance for the Optimal Assessment of Highway Structures”, ZAG Ljubljana, http://samaris.zag.si/, 2006. 
[4] L. Frýba, "Vibration of Solids and Structures Under Moving Loads", Noordhoff International Publishing, Groningen, The Netherlands, 1972.

[5] Danish Roads Directorate (DRD) Report 291, "Reliability-Based Classification of the Load Carrying Capacity of Existing Bridges", Roads Directorate, Ministry of Transport, Denmark, 2004.

[6] E.J. OBrien, A. González, J. Dowling, A. Žnidarič, "Direct measurement of dynamics in road bridges using a bridge weigh-in-motion system", The Baltic Journal of Road and Bridge Engineering, 11(4), 263-270, 2013.

[7] G. James, "Analysis of traffic load effects on railway bridges", $\mathrm{PhD}$ thesis, Royal Institute of Technology SE-100 44 Stockholm, Sweden, 2003.

[8] D. Cantero, E.J. OBrien, R. Karoumi, "Extending the Assessment Dynamic Ratio to Railway Bridges", in "Proceedings of the Second International Conference on Railway Technology: Research, Development and Maintenance", J. Pombo, (Editor). Civil-Comp Press, Stirlingshire, UK, Paper 67, 2014, doi:10.4203/ccp.104.67

[9] L. Connolly, D. Hajializadeh, C. Leahy, A. O'Connor, E.J. O'Brien, C. Bowe, "Calculation of the Dynamic Allowance for Railway Bridges from Direct Measurement", in J. Pombo, (Editor), "Proceedings of the Third International Conference on Railway Technology: Research, Development and Maintenance", Civil-Comp Press, Stirlingshire, UK, Paper 138, 2016. doi: $10.4203 /$ ccp. 110.138

[10] X. Hu, B. Wang, H. Ji, “A Wireless Sensor Network-Based Structural Health Monitoring System for Highway Bridges", Computer-Aided Civil and Infrastructure Engineering, 28, 193-209, 2013.

[11] J.P. Ou, "Some recent advances of structural health monitoring systems for civil infrastructure in mainland China", Structural Health Monitoring and Intelligent Infrastructure, Z.S. Wu and M. Abe (Editors), 131-144, 2003.

[12] M. Valinejadshoubi, A. Bagehi and O. Moselhi, "Structural Health Monitoring of Buildings and Infrastructure", International Journal of Civil, Environment, Structural, Construction and Architectural Engineering. 10 (6), 690-697, 2016.

[13] J.P. Lynch, K.J. Loh, "A summary review of wireless sensors and sensor networks for structural health monitoring", Shock and Vibration Digest, 38(2), 91-128, 2006.

[14] T.W. Parks, C. S. Burrus, "Digital Filter Design”, John Wiley \& Sons, Texas, United States, 1987.

[15] L.R. Rabiner, J.H. McClellan, T.W. Parks, "FIR Digital Filter Design Techniques Using Weighted Chebyshev Approximation", in "Proceedings of the IEEE" 63, 595-610, 1975.

[16] C. Britton Rorabaugh, "Digital filter designer's handbook: featuring C routines", McGraw-Hill Professional, 1993.

[17] BD 37/01 "Loads for Highway Bridges", in Design Manual for Roads and Bridges, Volume 1, Section 3, Part 14, Highways Agency, UK.

[18] BD 21/01 "The Assessment of Highway Bridges and Structures", in Design Manual for Roads and Bridges, Volume 3, Section 4, Part 3, Highways Agency, UK. 
[19] L. Connolly, A. O'Connor, E.J. OBrien, E.J., "Probabilistic modelling and assessment of railway bridges", $8^{\text {th }}$ International Conference on Bridge Maintenance, Safety \& Management, IABMAS2016, Foz do Iguaçu, Brazil, 2630 June, 2016.

[20] Midas Information Technology Co., Ltd. Midas Civil Available at: http://en.midasuser.com/product/civil_overview.asp [Accessed 10 August 2015]

[21] E. Castillo, "Extreme Value Theory in Engineering", Academic Press Inc., London, United Kingdom, 1988.

[22] S-K. Choi, V. Grandhi, R.A. Canfield, "Reliability-Based Structural Design", London: Springer, 2007.

[23] ISO 2394, "General Principles on Reliability for Structures", 1998.

[24] JCSS, Joint Committee of Structural Safety, "Probabilistic Model Code I Basis of Design", 2000.

[25] A. O'Connor, C. Pedersen, L. Gustavsson, I. Enevoldsen, "Probability based assessment and optimised maintenance planning for a large riveted truss railway bridge", Structural Engineering International. 19(4), 375 - 383, 2009.

[26] Kavitha, P. E. "Strain Transformation and Rosette Gage Theory." Georiga Institute of Technology School of Aerospace Engineering, AE3145 Laboratory 1-7: 2009.

[27] EN 1991-2, 2003, "Eurocode 1: Actions on structures - Part 2: Traffic loads on bridges", European Standard, CEN, Brussels, 2003.

[28] J. Leander, A. Andersson, R. Karoumi, "Monitoring and enhanced fatigue evaluation of a steel railway bridge." Engineering Structures 32.3: 854-863, 2010.

[29] E.J. OBrien, A. Bordallo-Ruiz, B. Enright, "Lifetime maximum load effects on short-span bridges subject to growing traffic volumes", Structural Safety, 50, 113-122, 2014.

[30] D.V. Lindley, "Introduction to Probability and Statistics from a Bayesian Viewpoint”, Part 2, Inference. CUP Archive, 2011. 